\title{
Nova espécie de Thaumastus da Floresta Atlântica do Paraná, Brasil (Mollusca, Gastropoda, Pulmonata, Bulimuloidea)
}

\author{
Eduardo Colley
}

Departamento de Zoologia, Programa de Pós-Graduação em Ciências Biológicas - Zoologia, Universidade Federal do Paraná, Caixa Postal 19020, 81531-990, Curitiba, PR, Brasil. (eduardocolley@yahoo.com.br)

\begin{abstract}
New species of Thaumastus from Atlantic Forest of Paraná, Brazil (Mollusca, Gastropoda, Pulmonata, Bulimuloidea). Thaumastus straubei sp. nov. is described from Atlantic Forest of state of Paraná, Brazil. The generic attribution came from morphological analysis of the shell, radula, jaw and soft parts showing unique and exclusive features that allow distinction from all others species of genus known so far.
\end{abstract}

KEYWORDS. Shell, jaw, taxonomy, biome, Neotropical.

RESUMO. Thaumastus straubei sp. nov. é descrita da Floresta Atlântica do Estado do Paraná, Brasil. A atribuição genérica baseou-se a partir de análise morfológica da concha, rádula, mandíbula e das partes moles, evidenciando características únicas e exclusivas que permitem a distinção das demais espécies do gênero conhecidas até o momento.

PALAVRAS-CHAVE. Concha, mandíbula, taxonomia, bioma, Neotropical.

A Floresta Atlântica representa um dos biomas mais importantes do mundo quanto à diversidade biológica e complexidade dos ecossistemas. Representa um dos complexos florestais mais ameaçados do planeta e integra a fauna mais ameaçada do Brasil (MMA, 2000). A malacofauna terrestre que ocorre nesta região apresenta elevado grau de endemismo (Simone, 1999) e, tendo em vista o longo período de degradação da Floresta Atlântica desde o início da colonização da América do Sul, muitas espécies devem estar sendo extintas antes de serem conhecidas (Cullen JR. et al., 2006). Segundo dados da ConservaÇão Internacional (2009), a Floresta Atlântica é um dos 25 hotspots da biodiversidade mundial. O bioma e seus ecossistemas associados abrangia originalmente uma área de $1.360 .000 \mathrm{~km}^{2}$, correspondendo a cerca de $16 \%$ do território brasileiro ao longo da costa, desde o Piauí até o Rio Grande do Sul. Contudo, atualmente encontra-se fragmentado e reduzido a menos de $8 \%$ de sua extensão original (CONSERVAÇ̃̃o INTERNACIONAL, 2009).

O gênero Thaumastus Martens, 1860 possui distribuição exclusivamente sul-americana, com registros de ocorrência na Venezuela, Equador, Peru, Brasil e Chile (ZILCH, 1960). Foi descrito primeiramente como subgênero de Bulimulus Leach, 1814, tendo como espécie-tipo Bulimulus hartwegi Pfeiffer, 1846. STREBEL (1910) descreveu seis subgêneros em Thaumastus com base na escultura da protoconcha. Os estudos da concha associados às partes moles foram realizados posteriormente por Breure (1979) e ZiLCh (1960). MARTENS (1860) caracterizou Thaumastus como tendo a concha imperfurada, cônica-globosa, perióstraco acinzentado a castanho, abertura arredondada-ovalada com diâmetro longitudinal irregular, eixo columelar variavelmente inclinado e pigmentado, perístoma simples, regular, pouco expandido e não ornamentado.
O táxon descrito no presente estudo foi observado em campo pela primeira vez pelo naturalista Fernando Costa Straube em outubro de 1985 durante o desenvolvimento de uma atividade de pesquisa ambiental na UHG (Usina Hidrelétrica de Guaricana). Nesta ocasião o pesquisador realizou anotações sobre o hábito e o hábitat do molusco em ambiente natural. A partir destas informações foi conduzida uma nova excursão em busca de exemplares do táxon na área da UHG para a realização da análise taxonomica. O presente trabalho visa contribuir com o conhecimento da malacofauna terrestre da Floresta Atlântica do Paraná e sua conservação, tendo por objetivo descrever uma nova espécie de Thaumastus.

\section{MATERIAL E MÉTODOS}

O material foi coletado na área do entorno da Usina Hidroelétrica de Guaricana (UHG), localizada na Serra dos Castelhanos, a Sudoeste da Serra do Mar paranaense $\left(25^{\circ} 45^{\prime} \mathrm{S}, 48^{\circ} 55^{\prime} \mathrm{W}\right)$, que inclui a Área de Proteção Ambiental de Guaratuba. A área é atravessada em toda sua extensão pelo rio Arraial e forma a represa da usina que está localizada no município de São José dos Pinhais, a 720 m a.n.m. (acima do nível do mar), enquanto a usina propriamente dita está localizada $5,7 \mathrm{~km}$ serra abaixo, no município de Guaratuba. A cobertura vegetal na área de coleta é representada pela FOD (Floresta Ombrófila Densa) com transição entre as formações vegetacionais FOD Submontana até $600 \mathrm{~m}$. a.n.m. e FOD Montana acima deste limite (RoderJAN et al., 2002). O clima característico é o "Cfa" (Subtropical úmido) sem estação seca, com índice de pluviosidade e umidade relativa do ar elevado (MAACK, 1981).

Os exemplares foram obtidos em janeiro de 2007, tendo em vista o período de maior precipitação 
e umidade na região (IAPAR, 2009) que favorece a atividade dos moluscos terrestres. O material estudado está depositado no Departamento de Invertebrados da Coleção de Moluscos do Museu Nacional, Universidade Federal do Rio de Janeiro (MNRJ).

Em laboratório, os indivíduos vivos foram acondicionados em recipientes plásticos com tampa contendo água para serem distendidos. Após 48 horas foram transferidos para recipientes contendo álcool $70 \%$ como meio de fixação. O material coletado foi analisado e comparado com as conchas e exemplares, incluindo material-tipo de Bulimuloidea depositado no MNRJ, MHNC (Museu de História Natural do Capão da Imbúia) e MZSP (Museu de Zoologia da Universidade de São Paulo) e por meio das descrições e ilustrações originais e fotografias dos tipos.

A análise da concha foi realizada quanto a forma, dimensões e contagem do número de voltas baseada nas definições de Burch (1962) e Thomé et al. (2006) e coloração segundo KuPPERs (1979). A morfologia e terminologia utilizada na análise morfológica seguiu Jurberg et al. (1988), Pena et al. (1996; 2005) e SALGADO \& Coelho (1995).

A dissecção e desenhos foram efetuados sob estereomicroscópio acoplado com câmara-clara. Os espécimes preservados em álcool $70 \%$ tiveram a parte mole extraída manualmente da concha. $\mathrm{O}$ teto da câmara palial foi rebatido através de um corte em "U" a partir do pneumóstoma rompendo-se a parte distal da glândula digestiva e do reto. Deste modo foi possível visualizar o sistema digestivo e reprodutor, os quais foram destacados da massa cefalopediosa e posteriormente desenhados separadamente. A rádula e mandíbula foram extraídas manualmente, imersas em $\mathrm{KOH}$ (hidróxido de potassio) a $10 \%$ por 48 horas, desidratadas e mantidas em álcool $70 \%$. A forma, disposição e contagem do número de dentes das rádulas e número de placas da mandíbula foi realizada sob estereomicroscópio. As imagens em M.E.V. (Microscópio Eletrônico de Varredura) JeolJSM-6390 (MNRJ) foram obtidas a partir da montagem padrão das rádulas e mandíbula sobre pinos específicos.

As conchas foram fotografadas com câmera digital.

São utilizadas as seguintes abreviaturas ao longo do texto: h, altura; ha, altura da abertura; w, largura; wa, largura da abertura.

\section{Thaumastus straubei sp. nov.}

(Figs. 1-9)

Material-tipo. Holótipo, BRASIL, Paraná, Guaratuba, Área de Proteção Ambiental de Guaratuba (Usina Hidrelétrica de Guaricana), 01.I.2007, E. Colley col. (MNRJ-11890); parátipos, 7 exemplares (um jovem), 7 conchas, mesmos dados do holótipo (MNRJ-11890).

Localidade-tipo. Usina Hidrelétrica de Guaricana, localizada entre os municípios de São José dos Pinhais e Guaratuba $\left(25^{\circ} 45^{\prime} \mathrm{S}, 48^{\circ} 55^{\prime} \mathrm{W}\right)$.
Etimologia. O nome do novo táxon é em homenagem ao ornitólogo e consultor ambiental Fernando Costa Straube (Hori Consultoria Ambiental), que indicou a localidade para a coleta dos exemplares utilizados neste estudo.

Diagnose. Concha cônica, 63/4 voltas, pouco convexas. Perióstraco castanho-escuro com faixa central única amarelada. Protoconcha com $3 \frac{1}{2}$ voltas, ápice proeminente com núcleo intruso; primeira volta lisa, seguida por linhas axiais pouco inclinadas. Rádula $(10,20,1,20,10)$; dente central tricúspide, com placa basal desenvolvida nas extremidades; dentes marginais bicúspides. Mandíbula com 15-17 placas axiais sobrepostas. Massa cefalopediosa verde-escura. Teto da câmara palial uniformemente escuro, com faixa clara centralizada. Sistema reprodutor com pênis longo e calibroso; músculo retrator longo. Bursa copulatrix ligada ao $1 / 4$ da base do oviduto livre. Massa bucal com ramo do músculo columelar formado inicialmente por três feixes musculares; saco da rádula longo e projetado externamente.

Descrição. Massa cefalopediosa verde escura. Concha (Figs 1-3) cônica ( $h=50-52,4 \mathrm{~mm} / \mathrm{w}=20,7$ $22 \mathrm{~mm}$ ); imperfurada, sólida, 53/4-63/4 voltas, pouco convexas. Sutura rasa com inclinação regular. Perióstraco castanho-escuro (KuPPERS, 1979; A99, M99, C99) com faixa central única amarelada a partir da $5^{\text {a }}$ volta até o limite com a abertura (Figs 1,2). Protoconcha com $3 \frac{1}{2}$ voltas, ápice proeminente com núcleo intruso; primeira volta lisa seguida por linhas axiais pouco inclinadas, dispostas regularmente sem intervalo; na terceira volta as linhas se tornam progressivamente mais desenvolvidas, podendo estar ausente próximo ao limite com a teleoconcha (Fig. 3). Teleoconcha com linhas de crescimento inclinadas e irregulares. Espira $(h=32,2-$ $37,7 \mathrm{~mm}$ ) longa e afilada. Volta corporal desenvolvida com a faixa central amarelada, nítida. Abertura semiovalada (ha=22,9-24,8 mm/wa13-13,3 mm). Lábio externo longo e convexo formando um arco único, sem limite com o lábio basal curto. Eixo columelar pouco inclinado e levemente torcido no contato com a margem parietal; espessura regular, coloração esbranquiçada. Margem parietal pouco convexa, delimitada ou não por cicatriz esbranquiçada. Perístoma delgado, com espessura geralmente uniforme.

Mandíbula (Fig. 4). Arqueada, 15-17 placas axiais sobrepostas e desenvolvidas, com estrias de crescimento horizontais irregulares. Rádula (Figs 5, 6). Aproximadamente 84 fileiras e arranjo $(10,20,1,20,10)$; dente central tricúspide, com placa basal desenvolvida nas extremidades, endocone proeminente com ectocones pequenos e arredondados; dentes laterais bicúspides com endocone desenvolvido e ectocone pequeno; dentes marginais bicúspides e menores do que os laterais, com cúspides arredondadas.

Sistema renopericardio (Fig. 7). Teto da câmara palial escuro com pigmentação uniformemente distribuída 


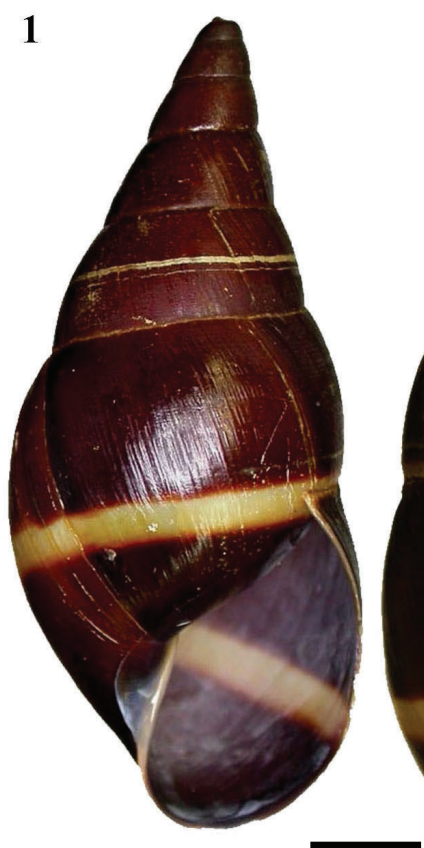

2

5

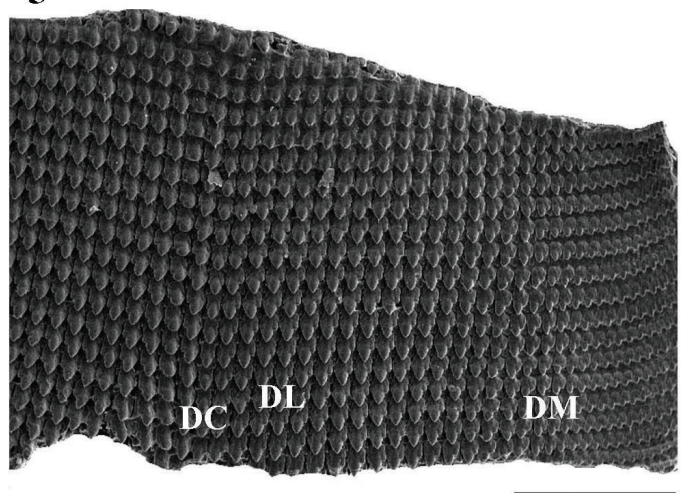

6

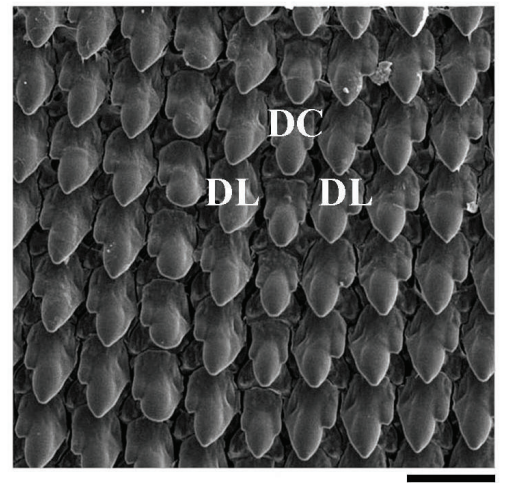

Figs 1-6. Thaumastus straubei sp. nov. Concha do holótipo: 1, vista ventral; 2, vista dorsal; 3, detalhe da protoconcha; 4, mandíbula; rádula: 5 ,

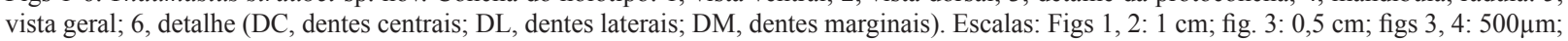
fig. 5: $100 \mu \mathrm{m}$.

com faixa clara centralizada (sem pigmentação) desde o pericárdio até o bordo do manto; superfície densamente preenchida por vasos sanguíneos que aparecem mais desenvolvidos na porção ad-retal e próximo ao pneumóstoma. Rim triangular, amplo, justaposto ao pericárdio. Ureter primário e secundário delgados, completamente fechados. Pericárdio alongado unido à longa veia pulmonar. Bordo do manto uniforme e delgado.

Sistema reprodutor (Fig. 8). Ovotestis constituído por inúmeros grupos foliculares irregulares, englobados pela glândula digestiva e ligados ao longo e enovelado ducto hermafrodita, que é mais desenvolvido próximo ao contato com o complexo de fertilização. Complexo de fertilização arredondado e desenvolvido, envolvido pela glândula de albúmen constituída por uma massa compacta de tonalidade clara, localizada no ápice do longo ovispermoduto, o qual é constituído quase que totalmente pelo útero esbranquiçado e pela próstata escura e folicular; na base da próstata emerge o ducto deferente que segue unido ao oviducto livre por fibras musculares e penetra no tegumento até alcançar o complexo peniano. Pênis longo, fibroso e calibroso; músculo retrator do pênis longo e desenvolvido, unido na porção apical do epipênis. Bursa copulatrix semiarredondada, ligada na porção do $1 / 4$ da base do oviduto livre por um longo ducto, mais desenvolvido entre a porção mediana até o contato com o oviduto. Vagina internalizada no tegumento.

Sistema digestivo (Fig. 9). Massa bucal arredondada, unida na base ao ramo do músculo columelar que é formado inicialmente por três feixes musculares; saco da rádula longo, projetado externamente para fora da massa bucal. Esôfago desenvolvido, porção mediana envolvida lateralmente pelo par de glândulas salivares dispostas irregularmente. Estômago amplo e saculiforme, aberto para o intestino. Glândula digestiva castanha-escura, foliculosa e rica em vasos. 
Hábito. Arbustivo; durante o repouso é encontrado preferencialmente na parte abaxial de folhas de arbustos localizados em ambiente com elevada umidade e solo pedregoso; quando em atividade também é observado se locomovendo sobre e entre rochas.

Discussão taxonômica. Thaumastus straubei sp. nov. apresenta características de concha, sistemas e estruturas exclusivos em relação às demais espécies conhecidas do gênero. Quanto ao aspecto geral da concha, se distingue por apresentar tamanho menor em relação ao mesmo número de voltas. A protoconcha apresenta maior número de voltas, sendo a primeira lisa seguida por linhas axiais pouco inclinadas até a terceira volta. A espira é afilada e o perióstraco castanho com faixa central amarelada. As espécies de Thaumastus conhecidas para o Brasil apresentam concha com dimensão maior, variando entre 55-95 mm de altura. O perióstraco em geral é de coloração castanho claro a escuro com mais de uma faixa clara dispostas no sentido axial ou subsutural como observado em T. achilles (Pfeiffer, 1852); T. ascendens (Pfeiffer, 1853); T. baixoguanduensis Pena et al., 1996; T. (T.) caetensis Pena et al., 2011; T. contortuplicatus (Reeve, 1850); T. lagillierti (Phillip, 1845); T. lundi Pena et al., 2005; T. magnificus (Grateloup, 1839); T. nehringi (Martens, 1839); T. (T.) parvus Pena et al., 2011; T. requieni (Pfeiffer, 1853); T. sellovii (King, 1831); T. spixii (Ferussac, 1821); T. strigatus (King, 1831); T. taunaisii (Férssac, 1822) e T. tiradentensis Pena et al., 1996 (Barros et al., 1991; Jurberg et al., 1988; Pena et al., 1996, 2005, 2011; SALGAdo et al., 1995; SALGado \& Coelho, 2003; Simone, 2006).

Thaumastus hebes (Strebel, 1910) é a única que
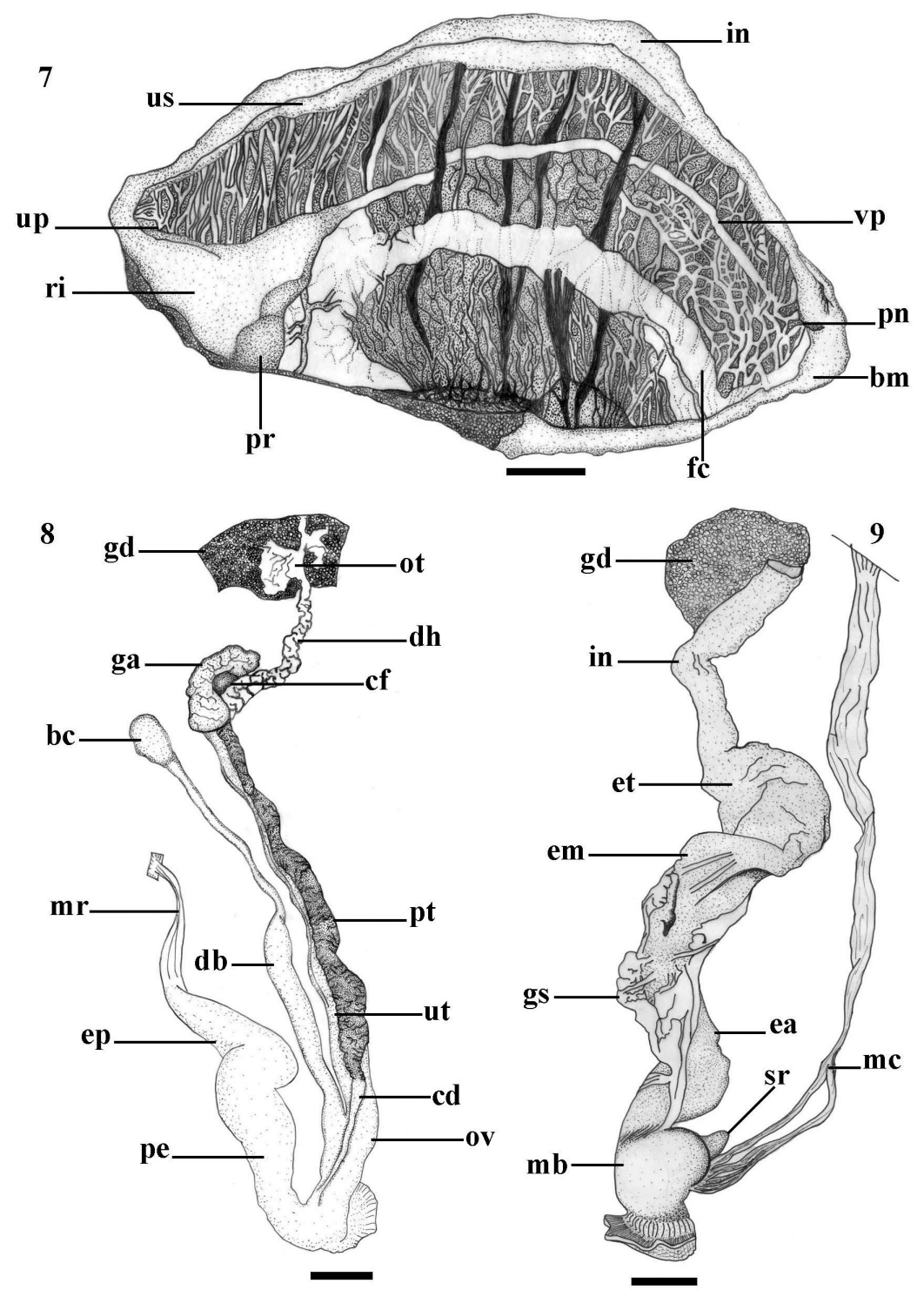

Figs 7-9. Thaumastus straubei sp. nov.: 7, teto da câmara palial; 8, sistema reprodutor; 9, sistema digestivo (bc, bursa copulatrix; bm, bordo do manto; cd, canal deferente; cf, complexo de fertilização; db, ducto da bursa copulatrix; dh, ducto hermafrodita; ea, esôfago anterior; em, esôfago médio; ep, epipênis; et, estômago; fc, faixa clara; ga, glândula de albúmem; gd, glândula digestiva; gs, glândula salivar; in, intestino; mb, massa bucal; mc, ramo do músculo columelar; mr, músculo retrator do pênis; ot, ovotestis; ov, oviduto; pe, pênis; pn, pneumóstoma; pr, pericárdio; pt, próstata; sr, saco da rádula; ri, rim; up, ureter primário; us, ureter secundário; ut, útero; vp, veia pulmonar). Escalas: $5 \mathrm{~mm}$ 
apresenta dimensão menor do que $T$. straubei sp. nov. $(\mathrm{h}=33 \mathrm{~mm})$, porém com o mesmo número de voltas e coloração distinta. Thaumastus baixoguandiensis possui concha com dimensão $\mathrm{h}=50-56,1 \mathrm{~mm} / \mathrm{w}=23-25,9$ $\mathrm{mm}$ e semelhante número de voltas, 6-61/4. Contudo, difere quanto à cor do perióstraco castanho-amarelada e protoconcha com estrias e pontuações (PENA et al., 1996). T. baixoguanduensis ainda se distingue do novo táxon por apresentar a rádula com dente central com cúspide mediana arredondada e dentes laterais e marginais com cúspides externas arredondadas; mandíbula com 11 placas e parte mole com a massa cefalopediosa castanho-acinzentada; teto da câmara palial distintamente vascularizado e sistema reprodutor com exteriorização do ducto deferente em posição inferior em relação ao oviduto livre (PENA et al., 1996). Entre as espécies de Thaumastus conhecidas para o Brasil, apenas T. achilles ocorre no Paraná (LANGE-DEMorretes, 1949; Salgado \& Coelho, 2003; Simone, 2006). O estudo do sistema reprodutor de T. achilles realizado por SALGADO et al. (1995) evidenciou a semelhança com $T$. straubei. Entretanto, além da dimensão da concha e coloração do perióstraco distintos, T. achilles, apresenta dentes da rádula com cúspides arredondadas e mandíbula com número inferior de placas (9-13) (Salgado \& Coelho, 1995). A diferença entre a área de ocorrência das duas espécies é outro fator que pode fortalecer a distinção entre T. achilles e o novo táxon proposto: a primeira se distribui por áreas de restinga (Langue-de-Morretes, 1949; Salgado et al., 1995), tecnicamente denominado de FOD das Terras Baixas, enquanto que T. straubei foi registrada apenas na encosta da Serra do Mar, representada pela FOD Submontana-Montana.

Agradecimentos. À equipe do laboratório de Malacologia do MNRJ, a Dra ${ }^{\mathrm{a}}$. Norma C. Salgado, Dr. Arnaldo C. S. Coelho e ao Dr. Alexandre D. Pimenta pelo acesso à coleção de moluscos; ao Dr. Paulo M. S. Costa pelo auxílio com a fotografia da concha e a Cláudio J. F. da Costa pelo auxílio com o material da coleção. Ao naturalista Fernando C. Straube pelas informações sobre a espécie. A Biól. Julia A. Santos (Departamento de Engenharia Ambiental/COPEL S/A) pelo parecer junto a COPEL e o acesso à área da usina. A Enga . Agrônoma Odete T. B. Carpanezzi (Departamento de Biodiversidade - IAP) pela autorização de coleta na APA de Guaratuba (IAP n ${ }^{\circ} 02 / 07$; IBAMA n¹89/2006). Aos responsáveis técnicos pelas coleções de moluscos, Dr. Luiz R. L. Simone (MZSP) e Biól. Sueli Kimiko (MHNC).

\section{REFERÊNCIAS BIBLIOGRÁFICAS}

Barros, H. M.; Coelho, A. S. \& Salgado, N. C. 1991. Superfamília Bulimuloidea do Brasil. Bulimulidae: Thaumastus (Thaumastus) magnificus (Grateloup, 1839) (Mollusca, Gastropoda, Pulmonata). Boletim do Museu Nacional, Zoologia 358:1-18.

BreURe, A. S. H. 1979. Systematics, phylogeny and zoogeography of Bulimulidae (Mollusca). Zoologische Verhandelingen (168):1215.

Burch, J. B. 1962. How to know the Eastern Land Snail. Dubuque, W. M. C. Brow Company Publishers. 214p.
Conservação Internacional (Brasil). 2009. Os desafios científicos para a conservação da biodiversidade no Brasil. Megadiversidade 5(1-2):1-144. Disponível em: <http://www.conservation.org.br/ publicacoes $>$. Acesso em: 03.06.2011.

Cullen Jr., L.; Rudran, R. \& Valladares-Pádua, C. orgs. 2006. Métodos de Estudo em Biologia da Conservação \& Manejo de Vida Silvestre. 2ed. Curitiba, Editora da UFPR/Fundação O Boticário. 651p.

IAPAR. 2009. Instituto Agronômico do Paraná. Resumos Históricos Climáticos - Sistema de Monitoramento Agroclimático do Paraná. Disponível em: <http://www.iapar.br/modules/conteudo/ conteudo.php?conteudo=1070>. Acesso em: 03.06.2011.

Jurberg, P.; Barros, H. M.; Gomes, L. A. L. \& Coelho, A. C. S. 1988. Superfamília Bulimuloidea do Brasil. Bulimulidae: Thaumastus (Thaumastus) taunaisii (Ferussac, 1822) com dados biológicos e aspectos comportamentais (Mollusca, Gastropoda, Pulmonata). Boletim do Museu Nacional, Zoologia 358:1-40.

KupPERs, H. 1979. Atlas de las cores. Barcelona, Blume. 161p.

Lange-De-Morretes, F. 1949. Ensaio de catálogo dos moluscos do Brasil. Arquivos do Museu Paranaense 7:1-216.

MaAck, R. 1981. Geografia Física do Estado do Paraná. 2ed. Curitiba, Secretaria da Cultura e do Esporte do Governo do Estado do Paraná. 442p.

Martens, E. 1860. In: Albers, J. C. \& Martens, E. Die heliceen nach naturlicher Verwandtschaft systematisch geordnet. Leipzig, Wilhelm Engelmann. 359p.

MMA- Ministério do Meio Ambiente. 2000. Avaliação e ações prioritárias para a conservação da biodiversidade da Mata Atlântica e Campos Sulinos. Brasília, Conservation International do Brasil, Fundação SOS Mata Atlântica, Fundação Biodiversitas, Instituto de Pesquisas Ecológicas, Secretaria do Meio Ambiente do Estado de São Paulo, SEMAD/Instituto Estadual de Florestas-MG. 40p.

Pena, M. S.; Salgado, N. C. \& Coelho, A. S. 1996. Bulimuloidea do Brasil. Duas novas espécies de Thaumastus Martens in Albers, 1860 (Mollusca, Gastropoda, Pulmonata). Boletim do Museu Nacional, Zoologia 358:1-12.

2005. New species of Thaumastus from Lagoa Santa region, Minas Gerais State, Brasil (Mollusca, Gastropoda, Pulmonata, Bulimuloidea). Iheringia, Série Zoologia 95(1):21-24.

.2011. Two new species of Tahumastus (Gastropoda: Pulmonata: Orthalicidae: Bulimulinae) from the state of Minas Gerais, Brazil. Zoologia 28(4):531-537.

Roderjan, C. V.; Galvão, F.; Kuniyoshi, Y. S. \& Hatschbach, G. G. 2002. As unidades fitogeográficas do Estado do Paraná. Ciência \& Ambiente 24:75-92.

Salgado, N. C. \& Coelho, A. S. 1995. Macroanatomia, microanatomia e histologia do sistema digestivo de Thaumastus (Thaumastus) taunaisii (Férussac, 1822), T. (T.) magnificus (Grateloup, 1839) e T. (T.) achilles (Pfeiffer, 1852). Boletim do Museu Nacional, Zoologia 362:1-19.

2003. Moluscos terrestres do Brasil (gastrópodes operculados ou não, exclusive Veronicellidae, Milacidae e Limacidae). Revista de Biologia Tropical 51(3):149-189.

Salgado, N. C. \& Coelho, A. S. \& Barros, H. M. 1995. Macroanatomia, microanatomia e histologia do sistema reprodutor de Thaumastus (Thaumastus) taunaisii (Férussac, 1822), T. (T.) magnificus (Grateloup, 1839) e T. (T.) achilles (Pfeiffer, 1852) (Mollusca, Gastropoda, Bulimulidae). Boletim do Museu Nacional, Zoologia 358:1-47.

Simone, L. R. L. 1999. Mollusca Terrestres. In: Brandẽo, C. R. \& Cancello, E. M. orgs. Biodiversidade do Estado de São Paulo, Brasil: Síntese do conhecimento ao final do século XX: Invertebrados Terrestres. São Paulo, Fapesp. v. 5, p. 3-8.

2006. Land and freshwater Molluses of Brazil. São Paulo, EGB, Fapesp. 390p.

Strebel, H. 1910. Conchologische Mitteilungen aus dem Naturhischen museum in Hamburg. Abhandlugem aus dem Gabiete der Naturwissenschaften 3:1-35.

Thomé, J. W.; Gomes, S. R. \& Picanço, J. B. 2006. Os caracóis e as lesmas dos nossos bosques e jardins. Pelotas, USEB. 123p.

Zilch, A. 1960. Euthyneura. In: Wenz, W. ed. Gastropoda. Berlin, Gebrud Borntrager. n. 2, 834p. 